\title{
Associations between skin barrier characteristics, skin conditions and health of aged nursing home residents: a multi- center prevalence and correlational study
}

\author{
Elisabeth Hahnel ${ }^{*}$ (D) Ulrike Blume-Peytavi, Carina Trojahn and Jan Kottner
}

\begin{abstract}
Background: Geriatric patients are affected by a range of skin conditions and dermatological diseases, functional limitations and chronic diseases. Skin problems are highly prevalent in elderly populations. Aim of this study was to investigate possible associations between health, functional and cutaneous variables in aged long-term care residents.

Methods: This observational, cross-sectional, descriptive prevalence study was conducted in a random sample of 10 institutional long-term care facilities in Berlin. In total, $n=223$ residents were included. Demographic and functional characteristics, xerosis cutis, incontinence associated dermatitis, pressure ulcers and skin tears were assessed. Stratum corneum hydration, transepidermal water loss, skin surface $\mathrm{pH}$ and skin temperature were measured. Data analysis was descriptive and explorative. To explore possible bivariate associations, a correlation matrix was created. The correlation matrix was also used to detect possible collinearity in the subsequent regression analyses.

Results: Mean age ( $n=223$ ) was 83.6 years, $67.7 \%$ were female. Most residents were affected by xerosis cutis (99.1\%; 95\% Cl: $97.7 \%$ - 100.0\%). The prevalence of pressure ulcers was $9.0 \%$ (95\% Cl: $5.0 \%$ - 13.0\%), of incontinence associated dermatitis 35.4\% (95\% Cl: 29.9\% - 42.2\%) and of skin tears $6.3 \%$ (95\% Cl: 3.2\% - 9.5\%). Biophysical skin parameters were not associated with overall care dependency, but with age and skin dryness. In general, skin dryness and measured skin barrier parameters were associated between arms and legs indicating similar overall skin characteristics of the residents.

Conclusion: Prevalence of xerosis cutis, pressure ulcers and skin tears were high, indicating the load of these adverse skin conditions in this population. Only few associations of demographic characteristics, skin barrier impairments and the occurrence of dry skin, pressure ulcers, skin tears and incontinence-associated dermatitis have been detected, that might limit the diagnostic value of skin barrier parameters in this population. Overall, the measured skin barrier parameters seem to have limited diagnostic value for the reported skin conditions except xerosis cutis.
\end{abstract}

Trial registration: This study is registered at https://clinicaltrials.gov/ct2/show/NCT02216526. Registration date: 8th November 2014.

Keywords: Dermatology, Nursing homes, Skin conditions, Biophysical measurements, Elderly

\footnotetext{
* Correspondence: Elisabeth.hahnel@charite.de

Department of Dermatology and Allergy, Clinical Research Center for Hair and Skin Science, Charité-Universitätsmedizin Berlin, Charitéplatz 1, 10117 Berlin, Germany
} 


\section{Background}

Geriatric patients are affected by a range of skin conditions and dermatological diseases. Pruritic dry skin (xerosis cutis) is the most common skin disorder in the aged with prevalence ranging from $5.4 \%$ to $85.5 \%$ [1-3]. In geriatric long-term care settings incontinence-associated dermatitis (IAD), skin tears, and pressure ulcers (PUs) are frequent $[4,5]$. The prevalence for IAD was reported to be 22.6\%, for skin tears 19.8\% [3] and for PUs up to 46\% [1]. Intrinsic age-related skin changes include elevated $\mathrm{pH}$, reduced stratum corneum turn-over rates, reduced stratum corneum hydration $(\mathrm{SCH})$ and reduced transepidermal water loss (TEWL) $[3,6,7]$. Extrinsic factors are functional limitations like immobility or incontinence leading to PUs [8] and IAD [9]. Empirical evidence suggests complex relationships between functional decline, age, cognition and the occurrence of adverse skin conditions [10-13]. For instance total Braden scale scores in patients aged 65 + years have been shown to be associated with the development of skin tears [13]. Kilic et al. showed associations between xerosis cutis and being bedridden [14]. An impaired skin barrier may be an indicator for higher susceptibility for skin disorders [3], hence biophysical skin barrier measurements (e.g. TEWL, $\mathrm{SCH}$ and $\mathrm{pH}$ ) are becoming more important for quantifying skin barrier characteristics in geriatric and long-term care research $[15,16]$. Aisen et al. proposed that skin hydration is reduced due to immobility in aged patients [17]. However, this finding was never reproduced. Associations and interactions between demographic, functional, clinical skin characteristics and skin barrier properties in geriatric patients have not been investigated systematically yet. We hypothesized that demographic characteristics, skin barrier impairments, and the occurrence of skin diseases in this vulnerable population are interrelated. Therefore, the aim of this study was to investigate possible associations between functional, skin barrier and cutaneous variables in aged long-term care residents and to investigate the strength and directions of these associations.

\section{Methods}

\section{Study design}

This was a descriptive, observational and cross-sectional study. The detailed description of the procedures are provided in the study protocol, which was published previously [18].

\section{Ethics approval and consent to participate}

This study was approved by the ethics committee of the Charité-Universitätsmedizin Berlin (EA1/190/14). Written informed consent was obtained from the residents themselves or their legal representatives prior any study procedure.

\section{Setting}

The study was conducted from September, 30th 2014 to March, 11th 2015 in 10 institutional long-term care facilities in Berlin. Using computer generated random numbers, nursing homes from a list of all existing nursing homes $(n=291)$ were contacted. In case of non-response the next randomly selected nursing home was invited.

\section{Participants}

The inclusion criteria were (1) resident of the respective nursing home, (2) aged $\geq 65$ years, and (3) written informed consent given personally or by legal representative. Residents at the end of life were not considered eligible.

\section{Variables}

Demographic variables like gender and age were collected. The Barthel-Index (BI) was used to measure physical function related to the daily activities with scores ranging from 0 (very care dependent) to 100 (not care dependent) [19]. Skin dryness was assessed with the Overall Dry Skin score (ODS) using a five-point scale ranging from '0' (no skin dryness) to '4' (advanced skin roughness, large scales, inflammation and cracks) [20, 21]. PUs were categorized according to the ICD 10 classification. The Braden scale was used to measure PU risk. Scores range from 6 (high PU risk) to 23 (no PU risk) [22]. IAD was classified according to the IAD-IT classification of Junkin 2008 [23] into four categories: early, moderate, severe, and fungal appearing rush, which may occur in addition to any category of IAD. Skin tears were recorded as present/absent. Cognitive function was measured with the Six Item Cognitive Impairment Test (6-CIT) [24]. Scores range from 0 (no sign of cognitive impairment) to 28 (significant cognitive impairment) [24, 25].

Biophysical skin measurements were conducted on intact skin at the right inner midvolar forearm and the right lateral lower leg. TEWL, $\mathrm{SCH}, \mathrm{pH}$ and temperature were measured using the non-invasive Multi Probe Adapter System MPA ${ }^{\bullet}$ (Courage \& Khazaka, Cologne, Germany) with Tewameter ${ }^{\circ} \mathrm{TM} 300$, Corneometer ${ }^{\circ} \mathrm{CM} 825$, Skin-pH-Meter ${ }^{\circ} \mathrm{PH} 905$ and Skin-ThermometerST500. All measurements were performed in triplicates. The arbitrary units (a.u.) for SCH measurement range from 0 to 120 , whereas higher readings indicate higher SCH. Elevated TEWL values indicate an increased evaporation of water molecules from the skin surface. Reference values of human skin $\mathrm{pH}$ range from 4 to $6[26,27]$. Skin surface temperature was measured in $\mathrm{C}^{\circ}$.

\section{Data sources and measurement}

All participating nursing home residents underwent a demographic, nursing, medical and dermatological examination. Among others, a full skin assessment was conducted by a dermatologist. Based on the possibilities 
of the institutions (e.g. availability of examination rooms) measurements were standardized as much as possible. However, optimal skin measurement conditions were not always achieved. Therefore, all procedures followed the guidelines for the in vivo measurement of TEWL and $\mathrm{SCH}$ in non-clinical settings [20]. The relative humidity (\%) and environmental temperature $\left(\mathrm{C}^{\circ}\right)$ was monitored throughout all skin measurements. Besides these two factors, the skin surface temperature is one of the most important predictor for TEWL [28]. Therefore, all TEWL estimates were converted to a standardized skin surface temperature of $30{ }^{\circ} \mathrm{C}$ according to the method by Mathias et al. [29].

\section{Bias}

Nursing homes were randomly selected from all nursing homes in Berlin to ensure generalizability. All study related procedures and measurements were conducted by trained dermatologists and study assistants according to standard operating procedures. All assessments and measurements were done using previously validated tools.

\section{Study size}

One aim was to measure the prevalence of PUs, IAD, skin tears, dry skin and to estimate skin barrier parameters. It was expected that the point estimates of proportions vary widely. Assuming a prevalence of 0.5 of skin diseases, approximately 280 residents would have been needed to measure this proportion with a desired width of a $95 \%$ CI of \pm 0.06 . According to the latest Nursing Care Statistics (2013), the size of the nursing home population in Berlin was approximately 30.000 [30]. Assuming 80 residents per institution and a participation rate of $50 \%(n=40)$ it was planned to include seven institutions which results in $n=280(7 \times \mathrm{n}=40)$ cases. All residents of the eligible nursing homes were invited, but participation rate was lower than $50 \%$. In order to reach the planned number, three additional nursing homes were recruited.

\section{Quantitative variables}

The sample was grouped according to gender and care dependency to take possible gender and care dependency differences into account. Care dependency was classified into mild to no dependency (total score 60-100), moderate (total score 20-59) and severe dependency (total score $0-19)$ based on the BI [31]. PU prevalence was reported for categories I to V and DTI (deep tissue injury) and excluding category I. An ODS of $\geq 1$ was categorized as xerosis cutis, relating to the five-point scale. Residents with sum scores $\geq 8$ according to the 6 -CIT were classified as 'cognitively impaired' [24].

\section{Statistical methods}

Data analysis was descriptive and explorative. Depending on the level of measurement (nominal, ordinal, continuous), demographic characteristics, clinical scores, skin conditions and skin biophysiological measurements were described using means, medians, proportions, frequencies and associated spread estimates. PU, skin tears, IAD and xerosis cutis prevalence was presented including 95\% confidence intervals $(\mathrm{CI})$. All variables were compared between gender and BI categories descriptively. To explore possible bivariate associations, a correlation matrix was created. Depending on level of measurement (e.g. metric and dichotomous) biserial or Pearson correlation coefficients were calculated. A minimum of $\geq 0.2$ or $\leq-0.2$ was considered as a minimum level of association. Based on the strengths and directions of associations and based on biophysiological considerations multivariable logistic and linear regression analyses were conducted. Special emphasis was put on possible associations between skin function, skin condition and skin care. Models were built iteratively to increase model fit indicated by Nagelkerke's $R^{2}$. The correlation matrix was also used to detect possible collinearity. The Durbin-Watson test and the variance inflation factors (VIF) were calculated to assess possible collinearity. A VIF of $>10$ and a Durbin-Watson test value $<1$ or $>3$ was regarded as indicative for autocorrelation.

\section{Results \\ Participants}

Fifty-five nursing homes were contacted. Ten nursing homes agreed to participate. Compared to participating institutions, non-participating were larger in terms of number of beds (mean beds per institution: 104.5 vs. $73.7)$ privately owned ( $76 \%$ vs. $60 \%)$ and non-profit $(30 \%$ vs. $22 \%)$.

In total, $n=811$ nursing home residents lived in the 10 nursing homes at the time of study visits. $N=252$ residents (31.1\%) provided written informed consent and $n=223$ were included (Fig. 1).

Groups of responders and non-responders of residents had comparable characteristics regarding gender, age and BMI (Table 1), indicating the external validity of the participants.

\section{Descriptive data}

Sample characteristics are shown in Table 2. Mean age was 83.6 (SD 8.0) years and $67.7 \%$ were female. Mean BMI was 25.3 (SD 5.1) $\mathrm{kg} / \mathrm{m}^{2}$. Mean Braden score was 17.3 (SD 3.7). $77.1 \%$ of the residents were cognitively impaired (6-CIT score $\geq 8$ ). The prevalence of xerosis cutis was $99.1 \%$ (95\% CI $96.8 \%$ to 99.8\%). Highest mean ODS scores were observed on both lower legs (2.1 (SD 1.0)) followed by the forearms (1.8 (SD 0.9)). Lowest mean ODS scores were graded on the trunk (1.3 (SD 0.8)). PU prevalence 


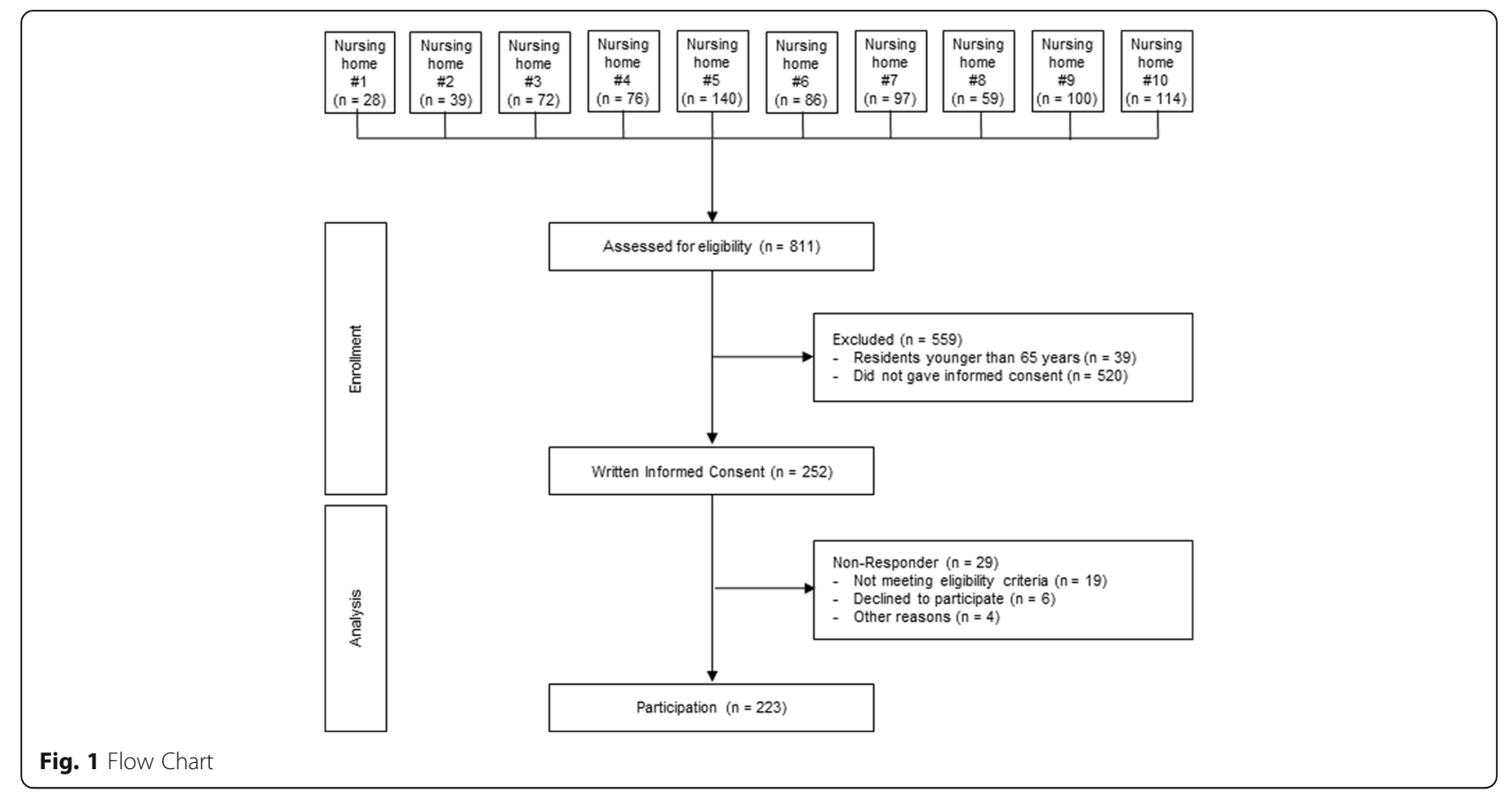

was $9.0 \%(95 \%$ CI $5.0 \%$ to $13.0 \%)$ and $3.6 \%$ (95\% CI $1.8 \%$ to $6.9 \%$ ) excluding category I. Most of the 20 PUs were located at the sacral region (45\%) followed by the heels $(25 \%)$ in residents with severe care dependency. Four PUs were observed at the back (20\%), one at the outer side of the left foot $(5 \%)$ and one at the plantar side of the foot (5\%). No category IV PUs and DTI were observed. IAD was diagnosed in 79 residents $(35.4 \%$, 95\% CI $29.9 \%$ to $42.2 \%)$. Most of the residents with IAD were male and had a moderate care dependency. Skin tears were present in 14 residents $(6.3 \%, 95 \%$ CI $3.2 \%$ to $9.5 \%)$ and were mostly located at the arms $(80 \%)$, followed by the legs $(20 \%)$. Skin tears on the legs only occurred in residents with severe care dependency. The temperature adjusted mean TEWL was 10.4 (SD 7.2$) \mathrm{g} / \mathrm{m}^{2} / \mathrm{h}$ on the midvolar forearm and $8.3(\mathrm{SD} 6.2) \mathrm{g} / \mathrm{m}^{2} / \mathrm{h}$ on the lower leg. Mean SCH was higher on the midvolar forearm (41.2 (SD 9.5) a.u.) than on the lower leg (34.5 (SD 10.2) a.u.). Mean pH

Table 1 Baseline data of responders and non-responders

\begin{tabular}{lll}
\hline & Responders $(n=223)$ & Non-Responders $(n=29)$ \\
\hline Female, n (\%) & $151 / 223(67.7)$ & $16 / 29(55.2)$ \\
Age [years] & & \\
Mean (SD) & $83.6(8.0)$ & $83.1(11.1)^{\mathrm{a}}$ \\
Median (IQR) & $84(78-89)$ & $81(73-95)^{\mathrm{a}}$ \\
BMl [kg/m $\left.{ }^{2}\right]$ & & $24.0(4.4)^{\mathrm{c}}$ \\
Mean (SD) & $25.3(5.1)^{\mathrm{b}}$ & $23.5(20.6-26.8)^{\mathrm{c}}$ \\
\hline
\end{tabular}

BMI Body mass index; ${ }^{\mathrm{a}} n=223 .{ }^{\mathrm{b}} n=216 .{ }^{\mathrm{c}} n=152$ values were comparable on both skin areas (5.4 (SD 0.6)). The mean room temperature was $22.9 \mathrm{C}^{\circ}$ (SD 1.5 $\mathrm{C}^{\circ}$; IQR $22.0 \mathrm{C}^{\circ}$ to $24.0 \mathrm{C}^{\circ}$ ) and the mean room humidity was $45.0 \%$ (SD 9.2\%; IQR 38.0\% to $52.0 \%$ ).

\section{Main results}

The strengths and directions of bivariate associations are shown in Table 3.

Age was most strongly associated with female gender $(r=0.285)$ and SCH on the lower legs $(r=0.205)$. The BI total score was associated with cognitive impairment $(r=-0.262)$, the Braden scale total score $(r=0.814)$ and a lower $\mathrm{pH}$ on the legs $(r=-0.204)$. The Braden total score was also associated with cognitive impairment of the residents $(r=-0.278)$ and lower $\mathrm{pH}$ on the legs $(r=-0.222)$. Intra-individual associations between TEWL, SCH and $\mathrm{pH}$ on the midvolar forearm and on the lower legs were high $(r=0.800 ; r=0.427 ; r=0.574)$. The skin surface temperature between the forearms and legs were associated as well $(r=0.522)$. A higher ODS on the arm was associated with a lower TEWL $(r=-0.228)$ and lower SCH values on the lower legs $(r=-0.242)$. Higher ODS on the lower leg was associated with decreased SCH $(r=-0.281)$. Skin dryness on the arms and the legs were strongly associated $(r=0.614)$. The occurrence of skin tears were associated with higher TEWL and SCH on the lower legs $(r=0.212 ; r=0.207)$. PU was associated with decreasing TEWL on the lower legs $(r=-0.209)$. 


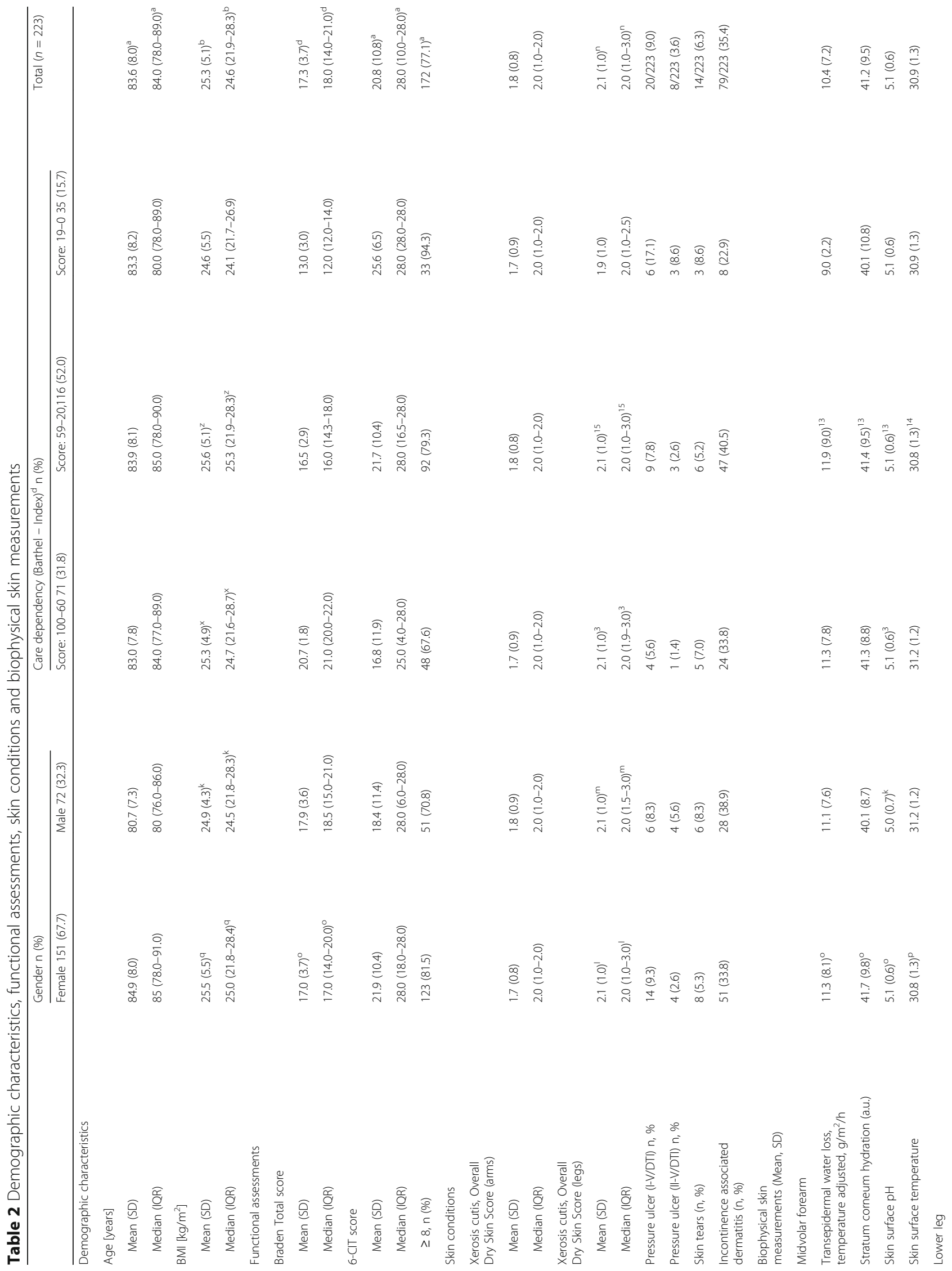




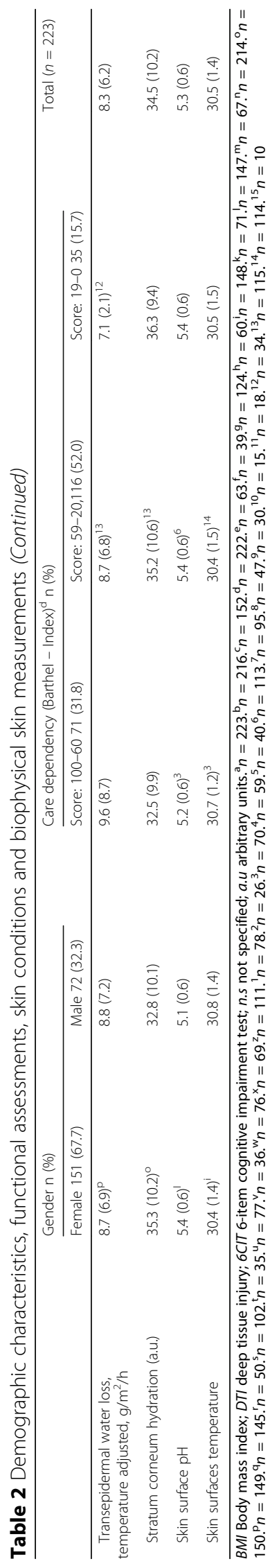




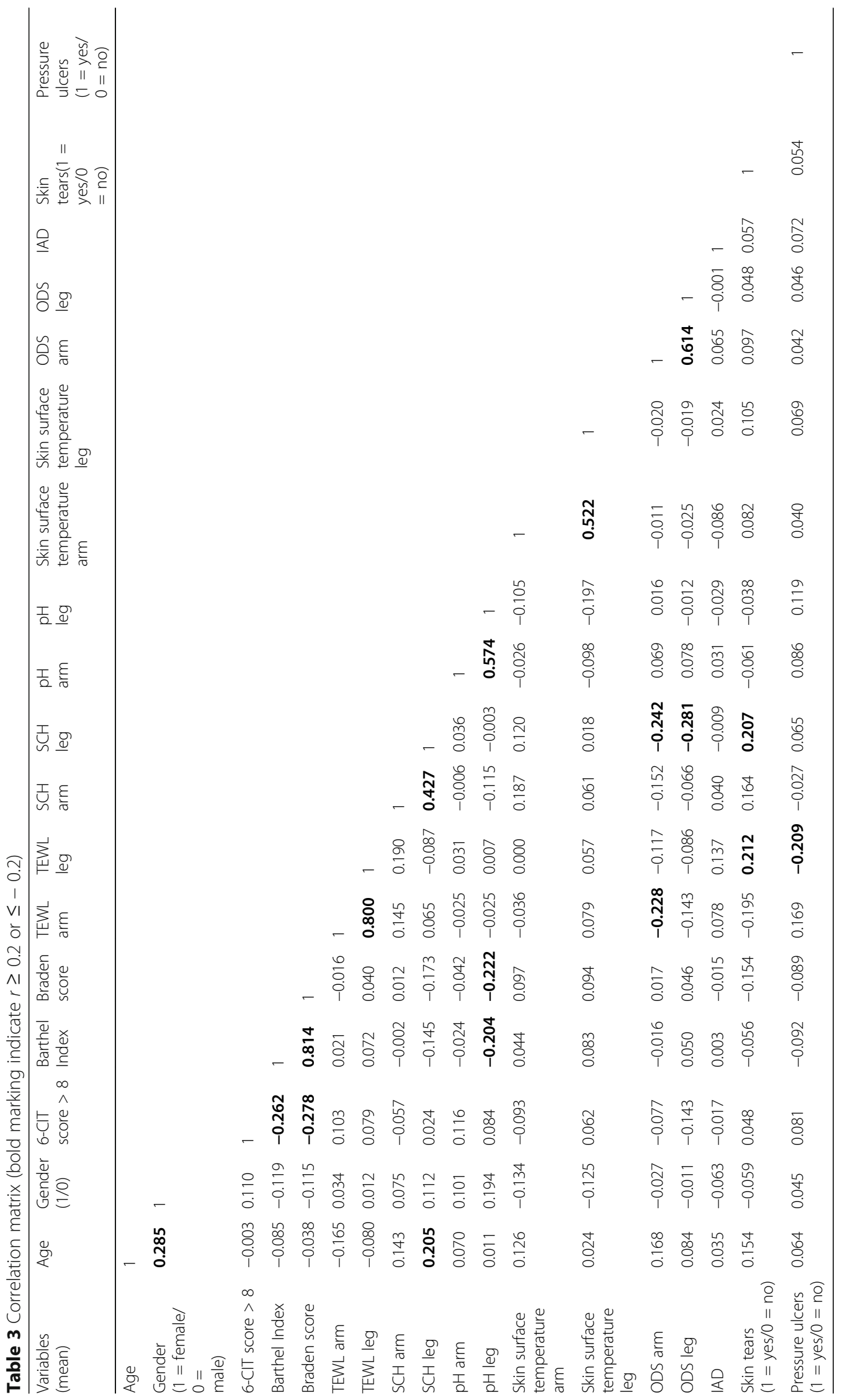


Table 4 Linear regression dependent variables: Skin barrier parameters

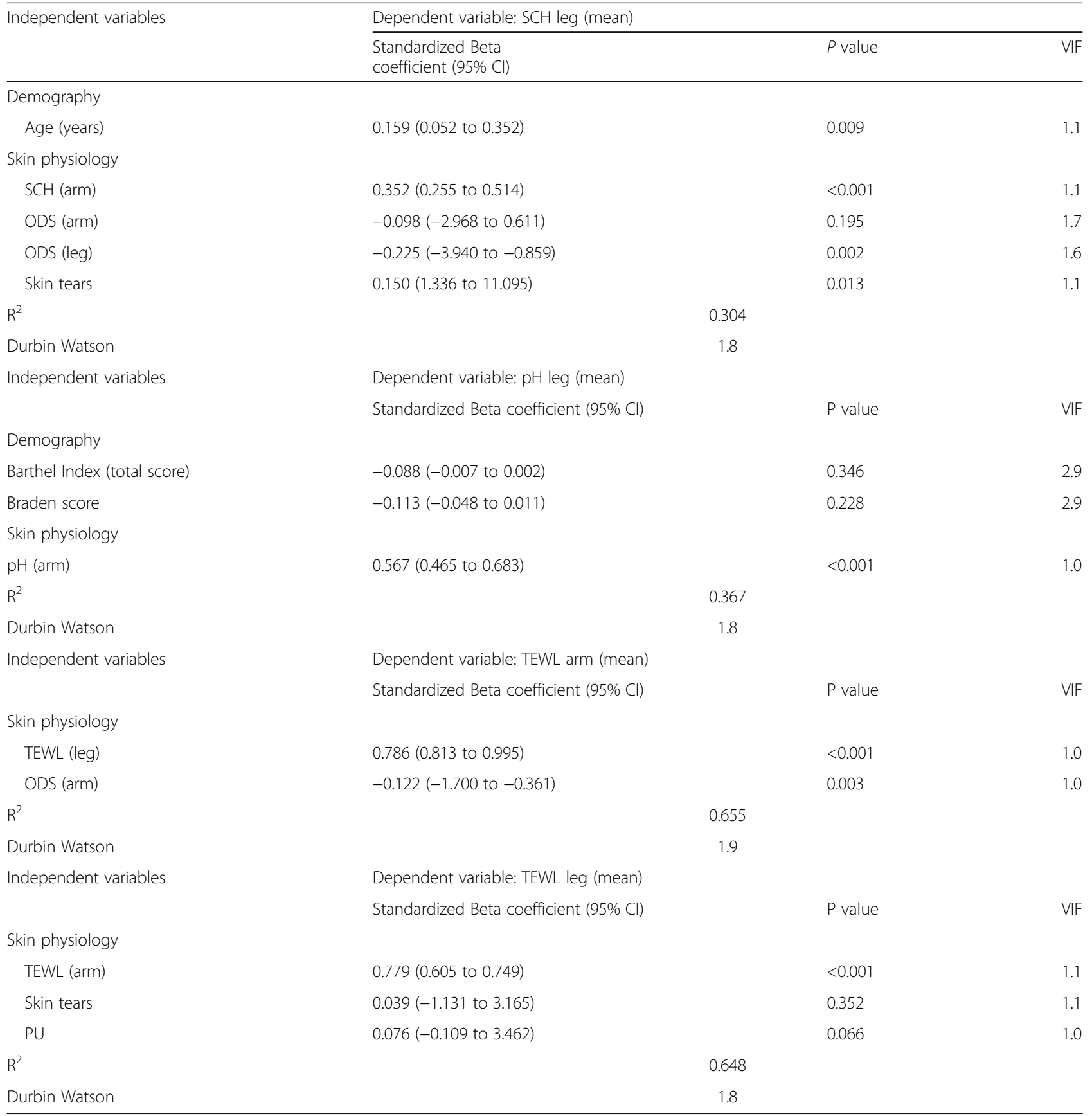

\section{Skin barrier parameters}

Based on the results of the bivariate associations, linear regression models were created. Results of linear regression models are displayed in Table 4.

A higher age was associated with increasing $\mathrm{SCH}$ on the lower legs $(\beta=0.159 ; p=0.009)$ and an increasing $\mathrm{SCH}$ on the midvolar forearms $(\beta=0.352 ; p<0.001)$. A lower ODS score $(\beta=-0.225 ; p=0.002)$ and the presence of skin tears $(\beta=-0.150 ; p=0.013)$ was associated with increasing $\mathrm{SCH}$ on the lower legs. A higher $\mathrm{pH}$ on the lower legs was associated with a higher $\mathrm{pH}$ on the midvolar forearms $(\beta=0.567 ; \mathrm{p}<0.001)$. The $\mathrm{BI}$ total score and the Braden score had no predictive ability in that model. A higher TEWL on the lower legs was strongly associated with higher TEWL values on the midvolar forearms $(\beta=0.786 ; \mathrm{p}=<0.001)$ and decreasing skin dryness on the midvolar forearms $(\beta=-0.122$, $p=0.003)$. Higher TEWL on the lower leg as dependent 
variable shows also a strong association with higher TEWL on the midvolar forearms $(\beta=0.779, p<0.001)$. The presence of skin tears and PU shows no predictive ability.

\section{Clinical outcomes}

Results of linear regression models, based on the results of the correlation matrix (Table 3) are displayed in Table 5 .

Increasing skin dryness on the arms was associated with decreasing TEWL on the midvolar forearms $(\beta=-0.203 ; p=0.002)$ and $\mathrm{SCH}$ on the lower legs $(\beta=-0.230 ; p=<0.001)$. Increasing skin dryness on the lower legs was associated with decreasing $\mathrm{SCH}$ on the lower legs $(\beta=-0.143 ; p=0.010)$ and increasing skin dryness on the midvolar forearms $(\beta=0.576$; $p<0.001)$.

\section{Discussion}

\section{Key results}

This cross-sectional study indicates that nearly every aged nursing home resident is affected by dry skin and more than one third suffered from IAD. In comparison with recently published studies in this setting the prevalences of PUs and skin tears of $9.0 \%$ and $6.3 \%$ were high $[13,32,33]$. For the first time, the three key skin barrier characteristics TEWL, SCH, and $\mathrm{pH}$ were measured in the German nursing home population. Overall, the number of associations was low. Strongest associations have been shown for the TEWL, SCH, pH, skin surface temperature and skin dryness between arms and legs indicating similar overall skin characteristics of the individual residents. Additionally, the functional parameters $\mathrm{BI}$ and Braden score were strongly associated with each other as well.

\section{Interpretation}

This was the largest randomly selected sample of nursing homes in which skin barrier measurements and clinical evaluations of different skin conditions have been performed. Study results indicate that nearly every aged nursing home resident is affected by dry skin (99.1\%). In comparison, this estimate is much higher compared to reported prevalences in this setting $[1,2]$. Leg skin was much drier than arm skin, which is supported by previous studies [2,34]. Clinical problems caused by dry skin include pruritus, which is also highly prevalent in geriatric patients [34, 35]. Pruritus induces scratching, leading to excoriations and enhances inflammatory reactions leading to secondary infection or superinfection. Ageing related loss of elasticity, dryness, atrophy and laxity of the skin can also lead to an increased skin susceptibility towards infections or skin damages, like PUs or IAD [7, 36]. Additionally, the prevalence of $35.4 \%$ of IAD and $8.5 \%$ of PU in our sample was also high compared to previously published studies $[1,32]$. The low number of associations being detected between biophysical measurements and clinical outcomes in aged long-term care residents indicate that these phenomena seem to be rather independent. Empirical evidence suggests, that there are associations between dry skin and PUs [32]. However, this finding could not be reproduced in this sample. The majority of skin tears was observed at the arms, but there was no association with $\mathrm{SCH}, \mathrm{pH}$, TEWL, or skin dryness.

Evidence further suggests that nursing home residents being more care independent are more likely to develop skin tears on the lower legs, whereas residents being more care dependent showed a higher occurrence of skin tears at the arms [10]. This association was not supported by our study results mainly because most skin

Table 5 Linear regression, dependent variables: Clinical outcomes

\begin{tabular}{|c|c|c|c|c|}
\hline \multirow[t]{2}{*}{ Independent variables } & \multicolumn{4}{|l|}{ Dependent variable: ODS arm } \\
\hline & Standardized Beta coefficient (95\% Cl) & & $P$ value & VIF \\
\hline \multicolumn{5}{|l|}{ Skin physiology } \\
\hline TEWL (arm) & $-0.203(-0.039$ to -0.009$)$ & & 0.002 & 1.0 \\
\hline $\mathrm{SCH}(\mathrm{leg})$ & $-0.230(-0.029$ to -0.009$)$ & & $<0.001$ & 1.0 \\
\hline$R^{2}$ & & 0.100 & & \\
\hline Durbin Watson & & 1.2 & & \\
\hline \multirow[t]{2}{*}{ Independent variables } & Dependent variable ODS leg & & & \\
\hline & Standardized Beta coefficient (95\% Cl) & & $P$ value & VIF \\
\hline \multicolumn{5}{|l|}{ Skin physiology } \\
\hline $\mathrm{SCH}(\mathrm{leg})$ & $-0.143(-0.024$ to -0.003$)$ & & 0.010 & 1.1 \\
\hline ODS (arm) & $0.576(0.529$ to 0.776$)$ & & $<0.001$ & 1.1 \\
\hline$R^{2}$ & \multicolumn{4}{|c|}{0.392} \\
\hline Durbin Watson & \multicolumn{4}{|c|}{1.3} \\
\hline
\end{tabular}

VIF Variance inflation factor 
tears occurred at the arms only. Irrespectively from these findings, recently published studies suggest improvement of xerosis cutis, skin tears as well as IAD by basic skin care interventions and structured skin care regimens [10, 37-39].

More than $77 \%$ of the sample was cognitive impaired. Any degree of cognitive impairment, indicated by 6-CIT score $>8$, was associated with lower BI and Braden scores. It is well known, that cognitively impaired nursing home residents are more likely affected by urinary incontinence, immobility and PUs compared to cognitively healthy residents [33]. Irrespectively, we were not able to show any associations between skin conditions and care dependency in our sample.

Only few recently published studies examining the associations of skin conditions and skin barrier measurements in nursing home residents exist, which limits the comparability of our results. Age-dependent changes in stratum corneum barrier function, TEWL and $\mathrm{pH}$ values are known $[15,40]$. The study of Aisen et al. showed a reduced skin hydration in prolonged immobile aged patients [17]. We could not reproduce this finding. Mean TEWL on the midvolar forearms and lower legs was higher compared to previous studies in this population $[16,41]$, indicating that TEWL is not an absolute value. Changes of TEWL between measurements over the time are more important for interpretation. On the other hand, $\mathrm{SCH}$ and $\mathrm{pH}$ values were similar to previous reports in this population $[16,42]$. In comparison, the $\mathrm{pH}$ was higher than in younger age groups $[15,43]$ indicating a less acidic skin in the elderly, which might affect the regulation of bacterial colonization and the desquamation process of the skin. In our study, lower $\mathrm{SCH}$ and TEWL are associated with increased skin dryness, which is in line with physiologically dry skin induced by aging or so called 'senile xerosis' [44]. This also indicates that a lower TEWL is certainly not an indicator for a clinical observed improvement in dry skin in this population [38]. Overall, the measured skin barrier parameters seem to have limited diagnostic value for the reported skin conditions except xerosis cutis.

\section{Limitations}

The anticipated sample size of $n=280$ was not achieved and there were differences between participating and non-participating institutions in terms of size and ownership. Whether this possible selection bias affected the results is unclear. Skin measurements were standardized as much as possible according to the circumstances in the nursing homes (e.g. monitoring of the room temperature and humidity, adjustment of TEWL to the skin surface temperature). All measurements followed the guidelines for the in vivo measurements in nonclinical settings. However, standardized and optimal conditions for the measurements were not achieved. Finally, skin barrier measurements were performed on arm and leg skin areas only. These area specific variables might not be relevant to cutaneous conditions at other skin areas, e.g. IAD.

\section{Generalizability}

Using a population-based approach and randomly selected nursing homes, $n=223$ aged nursing home residents were included. Facility characteristics were well comparable to all nursing homes in the federal state of Berlin, Germany in terms of sponsorship (privately owned $60 \%$ vs. $50.5 \%$; non-profit $30 \%$ vs. $45.2 \%$; public $10 \%$ vs. $4.2 \%$ ) or beds per institution (mean 73.7 vs.79.2) [45]. Demographic data like age, gender and care dependency are well comparable with the general German nursing home population statistics (e.g. females 67.7\% vs. $72.7 \%$; care-level I: $38.6 \%$ vs. $39 \%$; care-level II: $40.8 \%$ vs. $40.5 \%$; care-level III $18.4 \%$ vs. $21 \%$ ) [30] which supports the generalizability of the study results. Other characteristics like the mean Braden scale scores were comparable to previous studies in this population and setting, [46-48] indicating external validity of the study results.

\section{Conclusion}

Prevalence of xerosis cutis, PUs and skin tears were high, indicating the load of these adverse skin conditions in this population. Only few associations of demographic characteristics, skin barrier impairments and the occurrence of dry skin, PUs, skin tears and IAD have been detected, that might limit the diagnostic value of skin barrier parameters in this population. In general, the ODS and measured skin barrier parameters are highly associated between arms and legs indicating similar overall individual skin characteristics of the residents.

\section{Abbrevations}

AU: Arbitrary units.; Bl: Barthel-Index.; BMI: Body mass index.; Cl: Confidence interval.; CIT: Cognitive impairment test.; DTI: Deep tissue injury.;

IAD: Incontinence-associated dermatitis.; mL: Mili Litre.; ODS: Overall Dry Skin score.; OR: Odds ratio.; PU: Pressure ulcer.; SCH: Stratum corneum hydration.; SD: Standard deviation.; TEWL: Transepidermal water loss.; VIF: Variance inflation factor.

\section{Acknowledgements}

I, the corresponding author, confirm that I have listed all coauthors contributed significantly to the work. We would also like to thank all employees, who have contributed substantially to the success of this study. Our special thanks are directed to Dr. med Natalie Garcia Bartels, Dr. med. Gabor Dobos, Dr. med. Irina Jahnke and Dr. med. Vera Kanti regarding the dermatological examinations, medical and scientific advice as well as Dr. rer. medic. Andrea Lichterfeld-Kottner for critically reviewing the manuscript.

\section{Funding}

This investigator initiated clinical trial was supported by Galderma Pharma SA (Switzerland) and by the Clinical Research Center for Hair and Skin Science,

Department of Dermatology and Allergy, Charité - Universitätsmedizin Berlin. This had no influence on study design and data collection, study conduct, analysis and interpretation of data and in writing the manuscript. 


\section{Availability of data and materials}

The datasets used and/or analysed during the current study available from the corresponding author on reasonable request.

\section{Authors' contribution}

EH: Research associate and coordinator of the conducted study, substantial contributions to conception and design and acquisition, analysis and interpretation of data, preparation of manuscript. UBP: Substantial contributions to conception and design, dermatological examinations, preparation and review of the manuscript. CT: Research associate and coordinator of the conducted study, substantial contributions to conception and design, preparation and review of the manuscript. JK: Substantial contributions to conception and design, analysis and interpretation of data, preparation and review of the manuscript. All authors read and approved the final manuscript.

\section{Ethics approval and consent to participate}

The study was approved by the ethics committee of the CharitéUniversitätsmedizin Berlin (EA1/190/14). Written informed consent was obtained from the residents themselves or their legal representatives prior any study procedure. This study is registered at https://clinicaltrials.gov/ct2/ show/NCT02216526

\section{Consent for publication}

\section{Not Applicable.}

\section{Competing interests}

The authors declare that they have no competing interests and are alone responsible for the content and writing of the paper. The funding support of Galderma Pharma SA (Switzerland) had no influence on study planning, protocol preparation, study conduct, analyses and reporting.

\section{Publisher's Note}

Springer Nature remains neutral with regard to jurisdictional claims in published maps and institutional affiliations.

Received: 14 June 2017 Accepted: 1 November 2017 Published online: 13 November 2017

\section{References}

1. Hahnel E, Lichterfeld A, Blume-Peytavi U, Kottner J. The epidemiology of skin conditions in the aged: a systematic review. J Tissue Viability. 2016; doi: 10.1016/j.jtv.2016.04.001.

2. Lichterfeld A, Lahmann N, Blume-Peytavi U, Kottner J. Dry skin in nursing care receivers: a multi-centre cross-sectional prevalence study in hospitals and nursing homes. Int J Nurs Stud. 2016; doi:10.1016/j.jinurstu.2016.01.003

3. Lichterfeld A, Hahnel E, Blume-Peytavi U, Kottner J. Preventive skin care

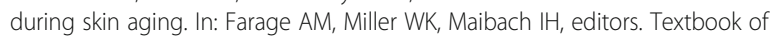
aging skin. Berlin, Heidelberg: Springer Berlin Heidelberg; 2014. p. 1-12. doi:10.1007/978-3-642-27814-3_133-1.

4. Kottner J, Balzer K, Dassen T, Heinze S. Pressure ulcers: a critical review of definitions and classifications. Ostomy Wound Manage. 2009:55:22-9.

5. Gray M, Black JM, Baharestani MM, Bliss DZ, Colwell JC, Goldberg M, et al. Moisture-associated skin damage: overview and pathophysiology. J Wound Ostomy Continence Nurs. 2011;38:233-41. doi:10.1097/WON. Ob013e318215f798.

6. Kottner J, Vogt A, Lichterfeld A, Blume-Peytavi U. Transepidermal water loss in young and aged healthy humans. In: Farage AM, Miller WK, Maibach $\|_{4}$ editors. Textbook of aging skin. Berlin, Heidelberg: Springer Berlin Heidelberg; 2014. p. 1-6. doi: 10.1007/978-3-642-27814-3_127-1.

7. Chang AL, Wong JW, Endo JO, Norman RA. Geriatric dermatology review: major changes in skin function in older patients and their contribution to common clinical challenges. J Am Med Dir Assoc. 2013;14:724-30. doi: 10.1016/j.jamda.2013.02.014.

8. Coleman S, Nixon J, Keen J, Wilson L, McGinnis E, Dealey C, et al. A new pressure ulcer conceptual framework. J Adv Nurs. 2014;70:2222-34. doi: 10.1111/jan.12405.

9. Beeckman D, Schoonhoven L, Verhaeghe S, Heyneman A, Defloor T. Prevention and treatment of incontinence-associated dermatitis: literature review. J Adv Nurs. 2009;65:1141-54. doi: 10.1111/j.1365-2648.2009.04986.x
10. Carville K, Leslie G, Osseiran-Moisson R, Newall N, Lewin G. The effectiveness of a twice-daily skin-moisturising regimen for reducing the incidence of skin tears. Int Wound J. 2014;11:446-53. doi: 10.1111/iwj.12326.

11. LeBlanc K, Kozell K, Martins L, Forest-Lalande L, Langlois M, Hill M. Is twicedaily skin moisturizing more effective than routine Care in the Prevention of skin tears in the elderly population? J Wound Ostomy Continence Nurs. 2016;43:17-22; quiz E11-12. doi: 10.1097/won.0000000000000195.

12. Rayner R, Carville K, Leslie G, Roberts P. A review of patient and skin characteristics associated with skin tears. J Wound Care. 2015:24:406-14. doi: 10.12968/jowc.2015.24.9.406

13. Sanada H, Nakagami G, Koyano Y, lizaka S, Sugama J. Incidence of skin tears in the extremities among elderly patients at a long-term medical facility in Japan: a prospective cohort study. Geriatr Gerontol Int. 2015;15:1058-63. doi: 10.1111/ggi.12405

14. Kilic A, Gul U, Aslan E, Soylu S. Dermatological findings in the senior population of nursing homes in Turkey. Arch Gerontol Geriatr. 2008;47: 93-8.

15. Sato N, Kitahara T, Fujimura T. Age-related changes of stratum corneum functions of skin on the trunk and the limbs. Skin Pharmacol Physiol. 2014; 27:181. doi: 10.1159/000353912.

16. lizaka S, Jiao L, Sugama J, Minematsu T, Oba M, Matsuo J, et al. Evaluation of nutritional status and skin condition among elderly residents in a longterm care hospital. J Nutr Health Aging. 2012;16:107-11.

17. Aisen E, Shafran A, Gilhar A. Sebum and water content in the skin of aged immobilized patients. Acta Derm Venereol. 1997;77:142-3.

18. Kottner J, Hahnel E, Trojahn C, Stroux A, Dobos G, Lichterfeld A, et al. A multi-center prevalence study and randomized controlled parallel-group pragmatic trial to compare the effectiveness of standardized skin care regimens on skin health in nursing home residents: a study protocol. Int J Nurs Stud. 2015;52:598-604. doi: 10.1016/j.jinurstu.2014.11.007.

19. Mahoney FI, Barthel DW. Functional evaluation: the Barthel index. Md State Med J. 1965:14:61-5.

20. Serup J. EEMCO guidance for the assessment of dry skin (xerosis) and ichtyosis: clinical scoring systems. Skin Res Technol. 1995;1:109-14.

21. Kang BC, Kim YE, Kim YJ, Chang MJ, Choi HD, Li K, et al. Optimizing EEMCO guidance for the assessment of dry skin (xerosis) for pharmacies. Skin Res Technol. 2014;20:87-91. doi: 10.1111/srt.12089.

22. Ayello EA, Braden B. How and why to do pressure ulcer risk assessment. Adv Skin Wound Care. 2002;15:125-31. quiz 132-133

23. Junkin J. Targeting incontinence-associated dermatitis. In: 3rd congress of the world Union of Wound Healing Societies; 2008.

24. Brooke P, Bullock R. Validation of a 6 item cognitive impairment test with a view to primary care usage. Int J Geriatr Psychiatry. 1999;14:936-40.

25. Abdel-Aziz K, Larner AJ. Six-item cognitive impairment test (6CIT): pragmatic diagnostic accuracy study for dementia and MCI. Int Psychogeriatr. 2015;27: 991-7. doi: 10.1017/s1041610214002932.

26. Lambers $\mathrm{H}$, Piessens $\mathrm{S}$, Bloem $\mathrm{A}$, Pronk $\mathrm{H}$, Finkel $\mathrm{P}$. Natural skin surface $\mathrm{pH}$ is on average below 5, which is beneficial for its resident flora. Int J Cosmet Sci. 2006:28:359-70. doi: 10.1111/j.1467-2494.2006.00344.x.

27. Schmid-Wendtner $\mathrm{MH}$, Korting $\mathrm{HC}$. The $\mathrm{pH}$ of the skin surface and its impact on the barrier function. Skin Pharmacol Physiol. 2006;19:296-302. doi: 10.1159/000094670

28. Rogiers $\mathrm{V}$. EEMCO guidance for the assessment of transepidermal water loss in cosmetic sciences. Skin Pharmacol Appl Ski Physiol. 2001;14:117-28. 56341

29. Mathias CG, Wilson DM, Maibach HI. Transepidermal water loss as a function of skin surface temperature. J Invest Dermatol. 1981;77:219-20.

30. Statistisches Bundesamt. Pflegestatistik 2013. Pflege im Rahmen der Pflegeversicherung Deutschlandergebnisse. Wiesbaden: Statistisches Bundesamt; 2015

31. McCusker J, Cole MG, Voyer P, Monette J, Champoux N, Ciampi A, et al. Sixmonth outcomes of co-occurring delirium, depression, and dementia in long-term care. J Am Geriatr Soc. 2014;62:2296-302. doi: 10.1111/jgs.13159.

32. Lechner A, Lahmann N, Neumann K, Blume-Peytavi U, Kottner J. Dry skin and pressure ulcer risk: a multi-center cross-sectional prevalence study in German hospitals and nursing homes. Int J Nurs Stud. 2017:73:63-9. doi: 10. 1016/j.jijnurstu.2017.05.011

33. Lahmann NA, Tannen A, Kuntz S, Raeder K, Schmitz G, Dassen T, et al. Mobility is the key! Trends and associations of common care problems in German long-term care facilities from 2008 to 2012. Int J Nurs Stud. 2015:52: 167-74. doi: 10.1016/j.ijnurstu.2014.07.014. 
34. Lichterfeld A, Lahmann N, Blume-Peytavi U, Kottner J. Dry skin in nursing care receivers: a multi-centre cross-sectional prevalence study in hospitals and nursing homes. Int J Nurs Stud. 2016;56:37-44. doi: 10.1016/j.jjnurstu. 2016.01.003.

35. Polat M, Yalcin B, Caliskan D, Alli N. Complete dermatological examination in the elderly: an exploratory study from an outpatient clinic in Turkey. Gerontology. 2009;55:58-63. doi: 10.1159/000129683.

36. Al-Nuaimi Y, Sherratt MJ, Griffiths CE. Skin health in older age. Maturitas. 2014;79:256-64. doi: 10.1016/j.maturitas.2014.08.005.

37. Brooks J, Cowdell F, Ersser SJ, Gardiner ED. Skin cleansing and emolliating for older people: a quasi-experimental pilot study. Int J Older People Nursing. 2017; doi: 10.1111/opn.12145.

38. Hahnel E, Blume-Peytavi U, Trojahn C, Dobos G, Stroux A, Garcia Bartels N, et al. The effectiveness of standardized skin care regimens on skin dryness in nursing home residents: a randomized controlled parallel-group pragmatic trial. Int J Nurs Stud. 2017;70:1-10. doi: 10.1016/j.ijnurstu.2017.02.006.

39. Kottner J, Lichterfeld A, Blume-Peytavi U. Maintaining skin integrity in the aged: a systematic review. Br J Dermatol. 2013;169:528-42. doi: 10.1111/bjd.12469.

40. Boireau-Adamezyk E, Baillet-Guffroy A, Stamatas GN. Age-dependent changes in stratum corneum barrier function. Skin Res Technol. 2014;20: 409-15. doi: 10.1111/srt.12132.

41. Kottner J, Lichterfeld A, Blume-Peytavi U. Transepidermal water loss in young and aged healthy humans: a systematic review and meta-analysis. Arch Dermatol Res. 2013;305:315-23. doi: 10.1007/s00403-012-1313-6.

42. Kottner J, Ludriksone L, Garcia Bartels N, Blume-Peytavi U. Do repeated skin barrier measurements influence each other's results? An explorative study. Skin Pharmacol Physiol. 2014;27:90-6. doi: 10.1159/000351882.

43. Marrakchi S, Maibach HI. Biophysical parameters of skin: map of human face, regional, and age-related differences. Contact Dermatitis. 2007;57:2834. doi: 10.1111/j.1600-0536.2007.01138.x.

44. Berardesca E, Maibach HI. Transepidermal water loss and skin surface hydration in the non invasive assessment of stratum corneum function. Derm Beruf Umwelt. 1990;38:50-3.

45. Statistisches Bundesamt. Pflegestatistik 2015. Pflege im Rahmen der Pflegeversicherung Landervergleich - Pflegeheime 2013. Wiesbaden. 2016.

46. Lahmann NA, Halfens RJ, Dassen T. Pressure ulcers in German nursing homes and acute care hospitals: prevalence, frequency, and ulcer characteristics. Ostomy Wound Manage. 2006;52:20-33.

47. Tannen A, Dassen T, Halfens R. Differences in prevalence of pressure ulcers between the Netherlands and Germany-associations between risk, prevention and occurrence of pressure ulcers in hospitals and nursing homes. J Clin Nurs. 2008;17:1237-44. doi: 10.1111/j.1365-2702.2007.02225.x.

48. Lahmann NA, Suhr R, Kuntz S, Kottner J. Over- and undersupply in home care: a representative multicenter correlational study. Aging Clin Exp Res. 2015;27:209-19. doi: 10.1007/s40520-014-0267-2.

\section{Submit your next manuscript to BioMed Central and we will help you at every step:}

- We accept pre-submission inquiries

- Our selector tool helps you to find the most relevant journal

- We provide round the clock customer support

- Convenient online submission

- Thorough peer review

- Inclusion in PubMed and all major indexing services

- Maximum visibility for your research

Submit your manuscript at www.biomedcentral.com/submit 